\title{
Michael Guihot and Lyria Bennett Moses (2020) Artificial Intelligence, Robots and the Law. LexisNexis Australia
}

\author{
Nicholas Korpela \\ Queensland University of Technology, Australia
}

\section{ISBN: 9780409349450}

When Tesla ${ }^{1}$ broadcasted their Artificial Intelligence (AI) Day on 20 August this year, they unveiled new plans to develop 'AI for General Purpose Robotics' or, more specifically, an android robot to carry out general tasks for its human owner. ${ }^{2}$ Elon Musk announced that the Tesla Bot will possess a suite of AI capabilities such as multi-cam video neural networks, neural net planning and dojo training. ${ }^{3}$ This particular project and its objectives should provide a sense of perspective when it comes to the increasingly ambitious and complex exponential nature of emerging technology.

With the above in mind, Guihot and Bennett Moses' book Artificial Intelligence, Robots, and the Law presents readers with timely insights into defining, understanding and reflecting on the legal approaches to this new technology. Their objectives were to examine not only how AI and robotics are purposed and deployed in society, but also the current issues resulting from this current wave of technological innovation. ${ }^{4}$ This includes efforts to pre-empt future problems and associated risks, and a proactive approach to using current law to protect legal rights and balance competing interests. However, if these rights cannot be sufficiently safeguarded as the law intended, the authors advocate for legal reform when the existing law becomes inadequate to guarantee those protections. ${ }^{5}$

Guihot and Bennett Moses contextualise the fundamental importance of humans as not only the driving force behind AI and robotics technology but inevitably the most affected by it as well. ${ }^{6}$ Likewise, it could be argued that these innovations will equally have an effect on various wildlife populations and ecosystems, particularly if such technology is used to accelerate things like deforestation, the overharvesting of natural resources and climate change. The authors also endeavour to dispel the common misconceptions and misunderstandings associated with AI. However, these so-called misunderstandings might seem deliberate at times, such as when opportunistic vendors falsely market their products as having AI capabilities, which further confuses consumers, lawyers, and regulators alike. ${ }^{7}$ It is presumably for this reason, among others, the authors deconstruct the

\footnotetext{
${ }^{1}$ Tesla are a company that manufactures electric cars, as well as solar and integrated renewable energy solutions for homes.

2 Tesla, "Tesla AI Day."

${ }^{3}$ Tesla, "Tesla AI Day."

${ }^{4}$ Guihot, Artificial Intelligence, Robotics, and the Law, 43.

${ }^{5}$ Guihot, Artificial Intelligence, Robotics, and the Law, 43.

${ }^{6}$ Guihot, Artificial Intelligence, Robotics, and the Law, 3.

${ }^{7}$ Guihot, Artificial Intelligence, Robotics, and the Law, 6.
} 
rather complex technological nature of the terminology for those who may be unfamiliar. This provides readers with a simpler way of conceiving and understanding the technology within the context of law, and the critical issues Guihot and Bennett Moses are seeking to clarify.

They similarly raise critically important questions on ethics and dedicate a chapter to discussing the principles that should be guiding the development and use of AI and robotics. In this regard, the authors observe that in the spirit of innovation, there might be a reluctance to constrain or otherwise anticipate how new technology could be used for an adverse purpose. ${ }^{8}$ While ethical rules are important, Guihot and Bennett Moses emphasise the enforcement of rights and fit-for-purpose regulatory frameworks should continue to be the paramount objective and standard. ${ }^{9}$ In this way they contend law reform does not always have to be reactionary to technology. ${ }^{10}$ Rather the intention of the law can be, and should be, constructed in a way that sufficiently captures any new iterations or adaptions of technology likely to interfere with another's legal interests. This would help provide public confidence in the law, as it then sustains resilience, uniformity, and a degree of certainty when enforcing what is fair and equitable. However, this approach would not be appropriate within the scope of current laws relating to big data and modern forms of surveillance, as those laws, in particular, require constant monitoring with regard to whether they remain fit for purpose. ${ }^{11}$

In summary, this book provides a comprehensive introduction into the world of AI and robotics, with detailed discussions on legal theory, as well as an overview of the current laws in Australia. The authors also examine and discuss legislation used in other jurisdictions, such as in the European Union. However, considering the many different topics covered by Guihot and Bennett Moses, this book is not a 'catch all' for those seeking more intricate and nuanced debates typically found in academic journal articles. Moreover, because this book was written with law students in mind, it may have benefitted from incorporating case studies or practice questions to test their knowledge further. Notwithstanding the above, this book remains a very suitable point of reference for both students and legal practitioners. I would equally recommend this book to the public who may be interested in how the law is developing with, and responding to, the current wave of technological innovation.

\section{Bibliography}

Guihot, Michael and Lyria Bennett Moses. Artificial Intelligence, Robotics, and the Law. LexisNexis Australia, 2020. Tesla. "Tesla AI Day." Streamed live 19 August 2021. Video, 3:03:20. https://www.youtube.com/watch?v=j0z4FweCy4M

\footnotetext{
${ }^{8}$ Guihot, Artificial Intelligence, Robotics, and the Law, 47.

${ }^{9}$ Guihot, Artificial Intelligence, Robotics, and the Law, 88.

${ }^{10}$ Guihot, Artificial Intelligence, Robotics, and the Law, 103.

${ }^{11}$ Guihot, Artificial Intelligence, Robotics, and the Law, 197.
} 